It is very generally admitted, that in Tetanus, few remedies afford greater relief than the warm bath; but, as ordinarily employed, its use can be continued for only a short time, and the frequent changes which it necessitates, are often injurious to the patient, by exciting the spasms or increasing their severity. To these objections the blanketbath is not exposed. If the wet blanket be not too much wrung out, and the water be very hot, and if the patient be well packed in dry blankets externally, the heat and moisture may be long retained, and he may lie for many hours undisturbed, and during this time, food, stimulants, and other remedies may be administered. The packing may also be made the means of administering anodynes through the medium of the skin.

During the period of nearly four years that I was connected with the Royal Free Hospital, I had uuder my care another case of Idiopathic Tetanus, which occurred in a man, forty-seven years of age, a labourer, who had taken cold while working in the fields. The attack was more acute and severe than that which I have just detailed : he was admitted on the third day of the disease, and died on the sixth. The treatment pursued consisted in the exhibition of antimony, calomel, and opium, with the warm bath. His death was probably accelerated by an attempt to introduce a tube down the csophagus, for the purpose of administering food. While resident in the Royal Infirmary of Edinburgh, I also witnessed a case of Idiopathic Tetunus, which proved fatal. I mention these cases, as Mr. Curling has stated that Idiopathic Tetanus is, in this climate, rarely a fatal disease. On the contrary, however, it will be seen, that of the three cases which have fallen under my personal observation, wne only has terminated favourably.

20, Finsbury Circus, December 1849.

\title{
FATAL CASE OF GASTRO-INTESTINAL HAMORRHAGE FROM CARDIAC DISEASE, WHILST UNDER THE INFLUENCE OF MERCURY.
} By W. WHITE COOPER, Eaq., F.R.C.S., Senior Surgeon to the North London
Infirmary for Diseases of the Eye.

Mr. V. S., a merchant from Havannah, consulted me on the 31 st October 1848. His statement was, that he had suffered, on several occasions, from severe febrile and liver attacks; and that, for some time previously, he had been annoyed with fits of vomiting of bile, pain in the right shoulder and side, etc. Whilst travelling on the continent, about two months before I saw him, he remarked that the flames of candles, when viewed with the left eye, appeared as blue as a Bengal light; he was teased with frequent musca and scintillations, pain over the brow, and headache; and objects appeared indistinct, as if seen through a film or mist.

On examination of the eye, the pupil was found to be contracted and nearly motionless, and the sclerotic had an unhealthy murky aspect; the largest type was discerned with great difficulty, and he could merely 
see the outline of my features His complexion was of a muddy yellow, tongue exceedingly loaded with thick yellow fur, and his breath horribly offensive. The abdomen was tense, and there was considerable tenderness and some fulness in the hepatic region; pulse 84, and irritable, and bowels confined. It was evident that there was active congestion, and probably chronic inflammation of the choroid and retina, and that the liver was also much congested : the first indication clearly was, to unload that viscus and empty the alimentary canal. Considering that, as he had lived in the tropics, he would require a tolerably active dose, I prescribed six grains of the chloride of mercury, to be followed, after the lapse of four hours, by a brisk aperient draught, and broth diet. A blister to be applied to the left temple. On the 2nd November, I saw my patient again, and finding that the bowels had not been acted on, I directed a draught of infusion of senna, with tincture of jalap and sulphate of magnesia, to be taken immediately, and repeated at the expiration of four hours, unless the bowels had been thoroughly unloaded. This second dose brought away a panful of foul offensive matter, and afforded much relief. On the 3rd inst. he was decidedly better; the tension of the abdomen had diminished considerably, and there was less tenderness in the right side and epigastrium. The gums, however, showed evidence of mercurial action, and there was some salivation. The usual washes of alum, etc., were prescribed, and a mild aperient mixture also, in order that an action on the bowels should be kept up.

November 4th. The general symptoms are greatly improved, and the indistinctness of vision diminished, he being able to discern letters more distinctly. The salivation, however, is profuse, and face much swelled. To wash the mouth frequently with weak brandy and water, to omit the aperient, which is no longer required, and to take a mixture of infusion of cascarilla and orange peel, with dilute mineral acid, every six hours. Diet, strong beef tea, isinglass jelly, etc.

November 5th. With the exception of the salivation, the improvement still continues. There is much swelling of the gums; and an ulcer, about the size of a split pea, has formed on the inside of the gum, on each side, corresponding to the last upper molar teeth, and from one of these ulcers there has been a trifling oozing of blood. I swept the gums with a solution of nitrate of silver. The medicine to be continued.

November 6th. The salivation less, but in the night there had been some bleeding from the mouth. Supposing it to have come from the ulcers, I again touched them with caustic, and desired that the mouth should be frequently rinsed with a gargle, containing tinct. matico, 3 iii ;

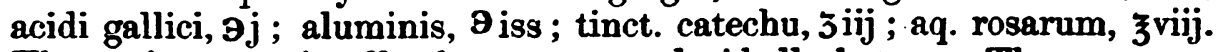
The patient was, in all other respects, decidedly better. There was no longer tenderness on pressure on the right side, the sight was improving, and he was cheerful. To take two grains of disulphate of quinine, with five drops of dilute sulphuric acid, every six hours, and to continue a bland nutritious diet.

November 7th. The salivation was nearly gone, and the patient felt so much better, that he went out for a drive for an hour. When I saw him in the evening, he said that he had spat a little blood; but I could not detect any bleeding spot, nor did it recur during an hour that I 
remained with him. He was desired to use the gargle frequently, and to continue the quinine.

November 8th. The sight steadily improves, and the patient considers himself convalescent. He has been out for another drive, and is making preparations for his departure for Cuba. At my evening visit, there had been no return of the bleeding; and I believed that it was at an end, little anticipating the catastrophe which was so shortly to follow.

November 9th. On my arrival at 11 A.M., I was grieved at finding a great change for the worse. Mr. V. S. had retired to bed on the previous evening in good spirits, and feeling very comfortable. About 11 P.M. he awoke with a sensation of suffocation; and, partly by coughing and partly by vomiting, relieved himself of a quantity of blood. He did not summon assistance; but continued at intervals, during the night, to havk up blood mixed with viscid saliva, and was greatly tormented with coagula in his throat, which he had difficulty in removing. I found him reclining on a sofa, pale, and still expectorating blood. This did not flow in gushes, nor pure, but was hawked up in the form of coagula, mixed with much viscid saliva. His pulse was rapid and feeble, and he was greatly depressed in spirits. I at once administered some hot brandy and water; and, when he had rallied a little, washed out the mouth with a sponge, and sought for the source of the hæmorrhage. With my fingers, I pulled out coagula from the fauces, but could not discover whence the blood came. Under these circumstances, and not desiring to take on myself the whole responsibility of the case, I sent for Mr. Fergusson, requesting him to bring instruments, in case it should be necessary to perform an operation. During the interval, I caused the patient to hold ice in his mouth, to gargle his throat with a strong styptic solution, and administered a dose of gallic acid. On Mr. Fergusson's arrival, he made a most careful examination, but with no better success than myself. In this state of absolute doubt as to the seat of the hæmorrhage, it was decided to give two grains of diacetate of lead, with half a grain of opium, every two hours; to support the strength by brandy in jelly; to cause the patient to swallow iced water frequently; and to carefully watch the symptoms. I remained with him several hours; and, at 11 P.M., had the satisfaction of finding that he had ceased to expectorate blood, and had fallen into a tranquil sleep at 9 P.M., having taken three of the pills. His bowels had been acted on thrice, and he had passed a considerable quantity of black grumous blood. Pulse 100, of very fair strength, and extremities warm. He was lying on a sofa on his right side; and, although he was still dressed, I did not think it prudent to disturb him by removal to bed. I sat up with him during the night, occasionally feeling his pulse and extremities. The former continued good, and the latter retained their heat. He continued to sleep in the same attitude until a little before 6 A.M., when, whilst I was standing watching him, he raised himself up, turned round, and laid down on his left side, with his head resting on his arm. Thinking that he was waking, I went up, and, placing my hand on his shoulder, inquired how he felt? Receiving no answer, I looked closely at his face, and saw at once that he was dead! Nevertheless, I tried all the means in my power to restore animation, but they were of no avail. He had fainted, and the heart had ceased to pulsate. 
A violent chill, from exposure to cold during my attendance on this case, confined me to bed for the two following days, and prevented my being present at the post-mortem examination, greatly to my disappointment. It was, howerer, conducted in the most careful manner, twenty-six hours after death, by Mr. Fergusson, to whom I am indebted for the following particulars:

"On inspecting the body of the late Mr. V. S. this morning, I concluded that the cause of death was bleeding from the bowels, complicated with disease of the heart. The intestines generally were filled with clotted blood, which had accumulated for many hours previous to death. The stomach contained the same; and the appearance of the mucous membrane of the canal indicated that it had been the seat of the hæmorrhage. The heart was at least one-third larger than natural, and its left ventricle was remarkably thickened. The liver was larger than natural. One kidney was somewhat diseased, but all the other viscera seemed tolerably healthy.

" There was no ulcer nor abrasion on the throat, fauces, or tongue ; and the bleeding from the mouth must have resulted from some peculiarity which had caused the escape of blood from the lining membrane of the stomach and bowels.

"Mr. V. S. was, as you know, salivated by a very small quantity of mercury. This condition is so common, while bleeding from the mucous membranes at the same time is so rare, that I cannot suppose the one to have been the result of the other.-W. F."

- Remarks. “ "Hæmorrhages", says Dr. Watson,' " from various parts of the mucous membranes, are, in truth, very common effects of cardiac disease"; and to this fact must the fatal result of the case before us be mainly ascribed. On review, it presents sereral points of considerable interest. Had the disease of the heart existed without mischief in the liver, or the liver been enlarged, the heart being sound, there would, in either case, have been a predisposition to intestinal hæmorrhage from the congestion caused in the abdominal vessels. The hyperæmia of the liver, in the present instance, would be a natural consequence of the cardiac disease; and thus there would result so great an obstruction in the abdominal circulation, as to gorge the vessels of the whole intestinal canal to the utmost, giving rise to hæmorrhage by exhalation from the mucous membrane. The spleen does not appear to have been enlarged to any extent, though such a condition might have been expected. Considering that the patient had been long resident in the Havannah (the climate of which is so pernicious to Europeans, that it is said that more than one-half of those who arrive there are carried off in the course of one year, principally by yellow fever), and that he had suffered from fever, it is matter of surprise there was not more extensive mischief in the liver and spleen.

The constitutional idiosyncracy, which led to the production of so powerful an effect from a single dose of calomel, could not have been foreseen, nor was the patient aware of such a peculiarity; but the mercurial action might have increased the hæmorrhagic disposition, by diminishing the cohesion of the blood, and lessening its power of coagula-

1 Loctures on the Practice of Physic, vol. i, 248. 
tion. Thus we find a series of conditions which, separately, might not have caused death at that time, but in combination, led to the fatal result. It is, however, clear that, with a heart so diseased, death must have taken place at no distant period.

A point which caused much embarrassment, and materially increased the obscurity of the case, was the mode in which the blood was got rid of. Instead of being vomited up, as might have been expected, considering the source whence its originated, it was havked up, as if it came from some part of the fauces or throat. The swelling from the mercurial action had not entirely subsided, rendering it difficult and painful to open the mouth widely; thus it was no easy matter to obtain a view of its interior: nevertheless, although neither $\mathbf{M r}$. Fergusson nor I could detect the seat of the bleeding, we satisfied ourselves that it was not in the mouth. It had occurred to me, that it might be a case of hæmorrhage by exhalation from the gums and fauces; but having directed the patient to cleanse those parts thoroughly by washing and gargling, I swept away the remaining coagula with a sponge, and carefully watched whether any blood exuded; nothing of the sort was visible, but after the lapse of a minute, the patient began to hawk and to endeavour to clear his throat of coagula. The distress occasioned by these clots was painful to witness; a mass would be drawn out, affording momentary relief, only to be followed by the tickling and choking sensation of another mass, and so firm were these, that it was necessary to scoop them out of the fauces with the finger, or to sweep them away with a sponge. During life, the impression on the minds of Mr. Fergusson and myself was, that the blood came from the fauces or their neighbourhood ; but the most careful examination after death failed to discover any grouud for this opinion; on the other hand, ample evidence appeared in the stomach and intestinal canal, especially the latter, that the blood had been poured out from the mucous membrane lining those parts.

The death of this patient illustrated extremely well the effect produced on a weakened heart by the erect posture being suddenly assumed. Well aware of the danger of throwing a burthen upon such a heart, I had particularly cautioned him against sitting up. To this he had attended whilst awake; but the stiffness, produced by lying the whole night on one side, probably caused him to shift his position whilst more than half asleep; he rose up with vigour, but the heart, unable to bear the additional column of blood, stopped, and life became at once extinct. The amount of blood lost was by no means such as to lead me to anticipate death; so far as could be judged, it was between four and five pounds-certainly not more; and so little did Mr. Fergusson expect a fatal result so soon, that he had made an appointment for the morrow, confidently believing that he should find an improvement in our patient, but he, poor man, died some hours before the time named for the consultation.

Tenterden Street, December 1849. 